\title{
Un atelier de langage en GS de maternelle peut-il préparer les élèves à entrer dans l'écrit?
}

\author{
Mireille Froment \\ Département de Sciences de l'Education, Université Paris Descartes \\ MoDyCo - Université Paris Descartes, CNRS, Université Paris Ouest Nanterre La Défense \\ mireille.froment@club-internet.fr
}

\section{Présentation et problématique}

Dans le milieu scolaire, le développement et l'appropriation des conduites langagières sont au cœur des préoccupations des praticiens et des didacticiens.

Les Instructions Officielles, depuis les années 90, mettent en avant, dès les premières années de la scolarité, la " maîtrise de la langue ${ }^{1}$ », présentée comme condition nécessaire à l'acquisition des savoirs scolaires. Des dispositions institutionnelles particulières concrétisent cette orientation, dès le niveau de la maternelle. C'est ainsi que la GS et la première année de l'école primaire (CP) sont intégrées dans un même cycle.

Bien que l'institution soit plus attentive qu'elle n'a pu l'être à l'enseignement de l'oral, l'écrit continue majoritairement à être considéré comme étant "naturellement» ce vers quoi doit tendre le travail de l'oral et le travail à l'oral. L'écrit visé par l'école est décrit comme permettant « d'outiller » la pensée, de par son caractère conservatoire, et également ses genres spécifiques (la liste et le tableau notamment). Les travaux de Goody (1977/1979), qui ont présenté l'écrit comme un facteur déterminant du développement des capacités cognitives, ont contribué à conforter cette accentuation, même si d'autres travaux (parmi lesquels des travaux ultérieurs de Goody, ceux de Lahire dans le domaine de l'école) se sont attachés à nuancer ce point de vue, voire à redéfinir et réévaluer les cultures orales.

L'ensemble de ces dispositions modifie les attentes scolaires, et ont des effets sur les pratiques. Actuellement, la GS de maternelle se trouve grandement orientée vers l'entrée dans l'écrit, ce qui conduit à importer dans les classes des modalités de travail propres au CP (notamment le travail "écrit» individuel), comme le remarquent -et s'en inquiètent, nombre de didacticiens (Bautier, Laparra, Brigaudiot...).

C'est à partir de ces évolutions et orientations que l'on soumet de nouveau à l'examen la problématique des rapports de continuité rupture entre oral et écrit.

Le présent article s'intéresse à un « atelier de langage » en GS de maternelle, qui s'est déroulé dans une classe unique, tout au long d'une année scolaire et s'est révélé très favorable au développement et à l'appropriation des conduites langagières.

Notre travail porte sur deux aspects que la problématique nous conduit à prendre ensemble : quelles conditions se sont trouvées réunies dans cet atelier de langage et ont favorisé le développement et l'appropriation de conduites langagières et en quoi cet atelier et ses conduites langagières orales peuvent préparer les élèves à entrer dans l'écrit.

\section{Introduction théorique}

A l'école maternelle, il ne s'agit pas d'apprendre à parler ${ }^{2}$ à un enfant, mais de l'aider à parler (François, 1993), de soutenir la parole de l'enfant (Nonnon 1996), de favoriser la parole des petits (Froment et Leber Marin, 2003). 
Ces particularités permettent de mieux cerner le sens de «oral». Rappelons-les pour plus de clarté 1) le langage oral s'acquiert dans le milieu familial, 2) il s'acquiert dans les contextes construits par les formats (ou scénarios) d'interaction, 3) le dialogue est «premier », d'abord au sens «d'échanges » -il est premier au plan du développement, et premier enfin, dans la perspective bakhtinienne du rapport dialogique, comme élaboration du sens dans l'enchaînement d'un discours sur un autre. Insister sur la primauté du dialogue ainsi défini c'est prendre en compte la circulation du sens, et porter attention aux significations construites dans le parler à plusieurs, au partage de l'espace discursif.

En milieu scolaire, les usages langagiers sont spécifiques. Il s'agit d'une part de développer les conduites langagières pour elles-mêmes, d'autre part de les développer en tant que support des apprentissages ultérieurs et dans ce cadre, c'est sur la relation écrit / savoir que l'on se concentre. La persistance de l'échec scolaire écrit conduit à se demander dans quelle mesure les activités orales peuvent avoir des effets sur l'écrit.

Ces préoccupations permettent d'expliquer l'intérêt renouvelé pour les relations entre conduites orales et conduites écrites.

Pour certains orthophonistes, linguistes, didacticiens et psychologues les difficultés rencontrées par les élèves à l'écrit révèleraient des difficultés également présentes à l'oral. Elles résulteraient d'un « rapport malheureux à l'oral » et plus largement au langage (Préneron, Meljac, et Netchine (1994), Pouëch (2001), Kugler 2000), l'échec à l'écrit serait « une conséquence ou un effet de l'échec à l'oral » (Halté, 2005).

Cela suppose d'envisager les rapports entre oral et écrit autrement que du seul point de vue des codes ou de différence des situations d'énonciation qui oppose co-présence de l'oral et communication différée de l'écrit et de ne pas considérer qu'oral et écrit sont dans une relation dichotomique, tout en reconnaissant la nécessité de tenir compte des spécificités de chacun d'eux.

La discontinuité la plus flagrante entre oral et écrit est l'absence du corps à l'écrit, notamment le regard, les gestes, les mimiques. A l'oral, il participe de l'élaboration des messages et de leur interprétation (François, Nonnon, Morel, Colletta). Par exemple, un geste, un sourire de connivence accompagnant un discours aident le récepteur à interpréter le message. Les signes non verbaux, les modulations intonatives sont porteuses de sens. Le corps est porteur de certaines significations non reprises par le langage, cette sémiotique corporelle est un mode de prise en compte de l'interlocuteur dans l'espace dialogique, elle contribue à la création d'un espace partagé. Les travaux de Colletta (2001), sur les récits oraux d'enfants jeunes dans le sens d'une approche éthologique mettent l'accent non sur les seuls énoncés mais aussi sur leurs contours prosodiques, leurs traits de vocalité, et les mouvements corporels (mimiques, gestes, changements de posture patterns oculaires).

Or l'une des difficultés de l'écrit réside justement dans l'absence de ce support et dans l'absence d'équivalence entre métasignes corporels et code écrit.

L'autre difficulté pour l'enfant qui entre dans la production d'écrit, liée à l'absence de la présence corporelle de l'autre est, du point de vue de la production, la modification qui affecte l'étayage. Savoir continuer « sous le regard» de l'adulte, du pair... est d'un autre ordre que savoir continuer seul dans la solitude de l'écrit.

Il est bien entendu que c'est là une différence essentielle entre oral et écrit. Les travaux de Fabre (1990) sur les brouillons d'écoliers ont montré que les jeunes enfants ont recours à l'usage de couleurs, de motifs graphiques (guirlandes, fleurs...), de variations de grosseur de caractères... Ces signes écrits non langagiers introduisent un aspect subjectif et peuvent être vus comme des éléments « valant pour» sinon une présence corporelle du moins l'évocation du corporel.

Néanmoins, rappelons que l'apparition d'un nouveau code n'est possible que s'il a une valeur symbolique, que s'il offre des potentialités qui ne se rencontrent pas dans un autre. Rappelons également que l'espace sémiologique est l'espace dans lequel peut s'acquérir le langage, d'abord oral puis écrit. Evoquer cet espace et le «dialogue » des sémiologies est une façon de pointer la possibilité de passer 
d'un code à l'autre, de traduire dans un code ce qui est perçu dans un autre, tout en reconnaissant que la spécificité du code ne peut pas ne pas affecter le sens.

Dans ces conditions, la question de savoir si l'atelier de langage peut préparer à l'entrée dans l'écrit nous conduit à reconsidérer la conception dichotomique de la relation oral / écrit. Des voies de recherche sont ouvertes, qui dépassent ce point de vue. La notion de conduite langagière théorisée par François (1993), reprise, continuée et retravaillée par d'autres chercheurs trouve un écho dans les travaux en didactique (Plane, Grandaty, Bucheton...). Halté (2005) défend une didactique "des activités langagières » qui prenne en compte le langage dans ses réalisations écrites et orales. Pour lui, l'échec de l'écrit est celui de l'oral, il est lié à une insuffisance du côté de l'enseignement de l'oral. Il en conclut qu'il faudrait développer une didactique de l'oral pour développer l'enseignement de l'écrit.

La notion de conduite langagière renvoie à la mise en œuvre de la langue par un sujet locuteur, dans une situation donnée, dans sa relation à un interlocuteur et un objet du monde. Il s'agit alors de langage (et non exclusivement de langue), de rapport dialogique (de sens à travers le mode d'enchaînement sur le discours de l'autre ou le sien propre), de jeux de langage.

La notion de conduite langagière permet de cerner, au-delà de la différence entre les codes, les processus langagiers qui leur sont communs et qui peuvent alors devenir des observables des situations d'enseignement apprentissage.

Avec la notion de conduite langagière l'attention se déplace de l'énoncé isolé vers le mode d'enchaînement d'un discours sur un autre discours, qui implique le mouvement discursif. Du point de vue bakhtinien de la «compréhension responsive » la façon dont un locuteur enchaîne sur le discours de l'autre (ou le sien propre) est porteur de l'interprétation compréhension de ce discours antérieur. Dans le dialogue, «une compréhension active contient l'ébauche d'une réponse (...) à chaque mot de l'énonciateur à décoder nous faisons correspondre une série de mots à nous formant une réplique » (Bakhtine, 1929/1977 : 146)

La notion de conduite langagière implique celle de genre, de monde et celle de place discursive. Les différentes relations du locuteur à l'objet du discours et à l'interlocuteur sont portées par le genre et le monde (ou univers de discours). Les objets dont on parle sont présentés à travers un genre, dans un monde qui peut être plutôt le monde du quotidien, de l'étrange, du souhaité... Le locuteur, qu'il le veuille ou non, ne peut pas ne pas donner une image de lui. Comme on l'a dit, cette notion de conduite langagière est indissociable de celle de la construction d'un sujet discursif.

Ces questions deviennent centrales quand on se place dans le cadre de l'enseignement-apprentissage et plus particulièrement l'enseignement-apprentissage adressé à de jeunes enfants.

La préoccupation essentielle devient alors celle du comment faire. En effet, les savoirs et savoir faire langagiers sont plutôt de nature empirique et s'acquièrent dans la pratique. On ne peut néanmoins, en conclure que toute action d'enseignement apprentissage serait sans effet, voire impossible.

Dans le cadre des situations aménagées par l'enseignant pour ses élèves, la notion de dispositif permet d'observer des paramètres de la situation et de mieux cerner les conditions qui favorisent le développement et l'appropriation des conduites langagières. Le dispositif est un médiateur à un double niveau, il a des effets sur la circulation du sens et à plus long terme sur le sujet.

Nous avons choisi de nous intéresser à un atelier de langage en maternelle, moment exclusivement consacré à la parole, en contraste avec d'autres situations où le langage est présent mais subordonné à une autre activité.

Notre observation analyse de l'atelier, entre caractéristiques générales qui ne dépendent pas de la situation scolaire et attentes de l'école, porte sur le dispositif spécifique de l'atelier, l'émergence des genres et les mouvements de subjectivation. Enfin, nous comparons l'évolution de deux enfants tout au long de ce dialogue. 


\section{Présentation du corpus}

Nous avons travaillé à partir d'un corpus constitué de 83 séances d'un atelier de langage ${ }^{3}$ qui se sont déroulées dans une école maternelle située dans une banlieue-quartier d'une ville française de province. L'étude longitudinale permet de mesurer l'importance du facteur temps en cernant des effets de la longue durée sur la dynamique du dispositif pédagogique et sur le devenir langagier des enfants, de chaque enfant.

A la rentrée de septembre, les 20 enfants de cette classe unique étaient âgés de 2,5 ans à 5,7 ans. L'atelier a été organisé à partir de la circulation d'une marionnette « Ploum », que chaque enfant, à tour de rôle, a emmenée à son domicile, à charge pour lui, le lendemain, de prendre la parole devant le groupe classe pour dire / raconter, expliciter ce qu'a fait Ploum / ce que lui-même a fait (aurait fait) avec la marionnette / au cours de la soirée. Chaque enfant de la classe a emmené 4 fois la marionnette chez lui, on a compté 4 Tours ${ }^{4}$, sur l'année.

L'atelier, mettant en place une situation peu contrainte, s'est montré propice à la diversité des voix, à un mode de participation diversifié de la part des enfants, autour d'un objet attrayant à la fois commun, singulier, présent et absent.

\section{Atelier de langage et dispositif}

En situation scolaire, certaines situations d'enseignement-apprentissage se montrent plus favorables que d'autres au travail cognitivo-langagier et à l'apprentissage. La notion de dispositif qui peut être rapprochée de celle de format d'interaction élaborée par Bruner, permet de prendre ensemble plusieurs paramètres qui construisent la situation d'enseignement et d'observer la façon dont ils interagissent.

Le dispositif n'est pas uniquement matériel, c'est aussi une manière de faire engageant un ensemble de relations, qui varient dans le temps (de la séance et/ou de la séquence), entre participants, matériel et objet de savoir. Le dispositif prévoit un objet discursif commun, un type de relation interlocutive, avec des rôles attribués. Il a une certaine durée et se répète à intervalles réguliers dans la vie de la classe si bien qu'il induit tout un système d'attentes mutuelles. Mais une situation aménagée n'est pas équivalente à une autre, son déroulement concret dépend de la thématique, des rôles et places discursives et de la tonalité des échanges.

Le dispositif de l'atelier de langage a permis, autour d'une marionnette (objet d'attention conjointe), de construire un espace d'attention conjointe.

Cet objet a favorisé l'engagement (ou investissement) des élèves (Goffman, 1981/1987) dans l'activité langagière. Durant tout le dialogue, les enfants ont eu de la marionnette une aperception ludique dont témoignent les moments d'effervescence, les joutes oratoires, l'augmentation des tours de parole en nombre et en longueur, les rires qui ont émaillé les séances. L'atmosphère ludique, le plaisir spécifique du parler à plusieurs ont aussi contribué à créer les conditions d'un rapport heureux au langage. L'objet d'attention conjointe a aussi offert la possibilité de maintenir et continuer un thème, de construire des relations de cohérence et de cohésion qui sont des exigences communes à l'oral et à l'écrit. (voir cidessous dialogue, genre et mouvements de genre)

La maîtresse a attribué des rôles, celui de locuteur principal à l'élève en charge de la marionnette qui devait rendre compte de (supposés) faits et gestes de la marionnette ayant eu lieu dans un autre espacetemps; celui de locuteurs auditeurs au reste de la classe. Puis dans un second temps les locuteurs auditeurs devenaient poseurs de questions adressées au locuteur principal. La maîtresse envisageait parallèlement un allègement progressif de ses propres interventions.

L'attribution de rôles était corrélée au déroulement séquentiel de la séance (à la première phase, phase d'installation, succède la phase où le locuteur principal dit ce qu'a fait la marionnette chez lui, puis la troisième où les auditeurs sont invités à devenir " poseurs de questions » et enfin la quatrième qui clôt la séance avec l'attribution de la marionnette à un autre élève). 
Le genre demandé était donc de raconter et de poser des questions afin de compléter le récit (du locuteur principal) ou bien de l'expliciter.

Il est bien entendu qu'un dispositif ne s'actualise pas tel qu'il a été pensé par l'enseignant. Son actualisation dépend de la façon dont la situation fait sens pour les participants, de l'enchaînement des discours, du rapport dialogique.

Ainsi, dans cet atelier, tout au long de l'année, les enfants ont appris à poser des questions visant à obtenir une information ou bien à faire une suggestion, ou bien encore à poser des questions portant sur le dit. Cette capacité a été inégalement partagée en fonction des âges, et dans le temps. Les plus grands sont davantage entrés dans cette conduite. Il n'y a cependant pas eu de coupure radicale entre petits et grands. Les plus grands comme les petits ont pu s'abandonner au plaisir spécifique du parler ensemble, ce dont ont témoigné les «bombardements de questions $5^{5}$ », au cours desquels ils ont rivalisé de rapidité et d'inventivité, sans vraiment attendre de réponse !

Une des stratégies dominantes des petits de 2/3ans ainsi que des 3/4ans a été de reprendre une assertion du locuteur principal pour en faire une question, ce qui constitue une façon de montrer la différence de place et constitue un degré dans cet apprentissage. Il a pu s'agir d'une reprise stricte ou bien d'une reprise de thème :

Ainsi quand Pauline (4/5 ans), locuteur principal, précise Ploum a mangé des carottes, il a bu, Lucile (2/3 ans) demande il a mangé ? il avait soif?

Les questions des $4 / 5$ ans et des $5 / 6$ ans peuvent amorcer un suivi du thème, une co-construction du discours. Ainsi quand Pauline (4/5 ans) interroge Arnauld ${ }^{6}$ (5/6 ans) :

PAU 1 - Arnauld! euh quand quand t'as fait du vélo t'étais avec ton papa?

ARN 8 -non! j'étais tout seul avec Ploum!

PAU 2 - dans la forêt?

ARN 9 -non!

La conduite de questionnement entre pairs peut jouer se concevoir comme une préparation à la conduite de monologue dans le dialogue: faire seul ce que l'on fait à deux. On ne parle pas cependant d'intériorisation, mais plutôt de possibilité d'occuper des places discursives différentes par rapport à l'interlocuteur et par rapport à soi.

Le dispositif de l'atelier de langage permet de faire des remarques importantes quant à la possibilité de ce dialogue de préparer à l'entrée dans l'écrit.

La dynamique de l'atelier de langage a mis les élèves en situation de parler de la marionnette, à partir de la marionnette, de rendre présents des objets, des événements, des personnes extérieurs à l'école, et à prendre en compte les savoirs et ignorances des interlocuteurs.

Dans l'atelier de langage, l'attribution de rôles interpelle l'élève dans une place discursive, pour parler d'un thème dans un genre, et lui donne ainsi la possibilité de diversifier ses conduites langagières. Ce n'est que progressivement que les élèves ont transformé ces rôles en place. Au plan de la séquentialité prévue par le dispositif, les phases «raconter ce qu'a fait la marionnette» et la phase "poser des questions » sont plus imbriquées que distinctes. A côté de ces rôles attribués, les élèves en ont souvent pris qui n'étaient pas suggérés préalablement, en fonction de la façon dont la situation a fait sens pour eux, en fonction des significations qui se sont élaborées tout au long du dialogue.

( $1^{\circ}$ Tour, séance 9 , Cécile, 3 ans, est locuteur principal)

\section{MAî 1 - alors: je sors Ploum du sac+ voilà PLOUM!}

CEC 1 - il est promené

MAÎ 2 - il s'est promené! 
NAB 1 - il est pas trop blanc/

CEC 2 - l'a mangé

THO 1 - il a mangé des ca(r)ottes

CEC 3 - mangé des carottes! a dormi

MAî 3 - il a dormi

CEC 4 - pis ap(r)ès il a mangé des pommes, ap(r)ès des fruits

MAÎ 4 - il n'a pas fait que manger ?

CEC 5 - non! il a pas tout mangé, après il a mangé à la table,

C'est le cas dans cet exemple, où l'on voit Thomas prendre la parole comme s'il était locuteur principal. Pour sa part, Nabil initie le genre commentaire dans le thème de Ploum.

Dans l'atelier de langage les élèves ont parlé d'un objet d'attention conjointe à la fois présent et absent, commun et différent. La diversité des modes de relation à l'objet (et plus particulièrement parler de l'objet absent) sont des conduites langagières qui peuvent se rencontrer aussi bien à l'oral qu'à l'écrit. Dans une situation d'écrit, le fait que l'interlocuteur ne partage pas l'espace-temps de l'énonciation rend nécessaire une représentation de la situation dont on parle et une mise en mots qui permette l'identification du référent .

Par la façon dont chaque locuteur se l'est appropriée, par ce qui lui est arrivé, par ce qu'elle a permis de dire, la marionnette a été ce par quoi s'est constituée une aire intermédiaire d'expérience (Winnicott $(1971 / 1975)$ dont le trait principal pour notre problématique est la rencontre sur un objet d'attention conjointe d'autres points de vue, la rencontre de l'altérité. Les enfants ont expérimenté la capacité d'être dans un espace transitionnel, de changer de «monde», (ou univers de discours) à multiplier discursivement les points de vue sur un même objet. Pouëch (2001: 21) insiste sur ce point. « L'apprentissage de l'écrit, écrit-elle, est lui aussi dans un espace potentiel entre un domaine où il n'y a rien d'autre que le soi et sa subjectivité et un domaine où il y a des objets et des phénomènes qui échappent au contrôle de soi ».

\section{Dialogue, genre et mouvements de genre}

La longue durée du dialogue autour de la marionnette permet de mieux comprendre comment émergent les genres comment s'articulent les genres autour d'un thème.

Dans un premier temps, les enfants ont d'abord « raconté », ce que la marionnette avait fait en enchaînant des énoncés constitués de prédicats biologiques et ludiques (il a fait dodo, il a mangé), actualisant $a$ plusieurs (dans la succession des tours de parole) le genre de la liste, cohérente de par le thème fédérateur. Au cours du déroulement des échanges, genre et thème ont évolué en même temps. Chaque locuteur a spécifié les actions énoncées, chaque locuteur l'a fait à sa façon, soit en modifiant l'objet sur lequel s'exerce l'action, ou encore le lieu

\section{Manger des carottes / de la pizza / des souris qui sontaient mortes \\ Il a fait dodo dans son lit / sur le canapé}

Soit en introduisant de nouveaux thèmes : La marionnette va à l'école, la marionnette fait des bêtises, du sport, part en vacances...

Une évolution notable (au $2^{\circ}$ Tour) est la mise en mots de processus qui induisent la temporalité du devenir. (Ploum va grandir)

La relation à la marionnette évolue: la spécification des actions et des processus contribuent à « construire » pour le locuteur un Ploum singulier. Suscitant d'abord des énoncés conflictuels quant à « son espèce» (l'assimilation de Ploum à un «hérisson» a provoqué de vives réactions au sein du 
groupe), c'est ensuite la tension entre perception effective (l'objet présent) et perception subjective qui a été au centre des discussions. Ploum (tout en étant une marionnette) a pu être vu comme un copain, un partenaire de jeu, un petit écolier, un sportif accompli... A partir du moment où à la relation «Ploumdoudou », s'ajoute la relation « Ploum-alter ego », s'est ouverte la possibilité de projeter dans le discours des moments de l'expérience propre.

Dans ce premier extrait, Nabil présente Ploum comme s'il était un compagnon de jeu, partageant les activités de l'enfant :

( $2^{\circ}$ Tour, Nabil, 5,11ans, est locuteur principal)

NAB 25 - il a beaucoup joué

MAî 49 - ah bon!

NAB 26 - il (a) été r(e)garder à la piscine mon frère et ma soeur et moi aussi! moi j'étais tout seul le mardi j(e) suis toujours tout seul à la piscine. il était au marché

MAÎ 50 - ah oui c'était le marché hier! $<3$ s.>

NAB 27 - on a ach(e)té des épinards

MÂ̂ 51 - aha!

NAB 28 - et d(e) la salade! \{brouhaha : on entend des voix\} y a un copain au marché eh ben il lui a donné un briquet

Dans ce second exemple, Pauline à travers le personnage de Ploum évoque des événements de son expérience propre.

( $2^{\circ}$ Tour, Pauline, 4,3 ans, est locuteur principal)

MAÎ 53 - où est-ce que tu voulais emmener Ploum, ma chérie?

PAU 17 - à l'hôpital

MAî 54 - ben oui! Pauline voulait emmener Ploum à l'hôpital:

PAU 18 - et pis j'l'ai oublié

MAî 54 - et puis tu l'as oublié!

NAB 5 - pourquoi elle voulait l'emmener?

MAÎ 55 - ah : oui! pourquoi elle voulait l'emmener? pourquoi? chut!

PAU 19 - <son papa est hospitalisé > pasque mon père il entend plus al(ors) faut pas crier!

MAî 56 - il entend plus, faut pas crier, et il est où ton père? \{on entend des chuchotements\}

PAU 20 - ben ! à son hôpital !

MAî 57 - oui:: \{on entend une voix, inaudible\}à quel hôpital il est? \{on entend des toux, des voix\} et tu voulais lui amener $\S$

FRA 1 - Ploum!

MAî 58 - oui, Ploum

FRA 2 - a [vaty]! à toto !§

Les reprises de la maîtresse, ses questions et celles de Nabil constituent un étayage pour continuer, expanser le thème et expliciter le lien entre Ploum et la visite à l'hôpital. Ce faisant, apparaît une des formes du monologue dans le dialogue répertoriée dans notre atelier. 
A l'écrit, le sujet manifeste une certaine « autonomie discursive » dans la mesure où il sait continuer seul. Dans l'atelier, le monologue dans le dialogue n'a été le fait que d'un petit nombre d'enfants, et diversement selon l'âge et le moment de l'année. Soit comme un «parler pour soi-même », souvent mal relié au contexte discursif, dont l'adresse est incertaine, soit comme monologue co-piloté par le locuteur soutenu par la maîtresse, qui dispense encouragements physiques et vocaux, soit enfin comme monologue compris dans un tour de parole, où le locuteur continue seul, en introduisant de nouveaux thèmes, de nouvelles catégories.

Au cours des $3^{\circ}$ et $4^{\circ}$ Tours les actions et processus continuent de se diversifier, mais surtout le thème connaît une évolution importante : la marionnette n'est plus aussi centrale, parler à partir de Ploum prend le pas sur parler de Ploum. Les genres évoluent. Le changement de thème modifie le genre et le monde (monde quotidien de la famille, monde de la fiction). Au cours de ces deux Tours, nous observons que les enfants interviennent plus fréquemment dans la prise en charge de la gestion du dialogue, mais surtout apparaissent des mouvements réflexifs, sous forme de commentaires du discours de l'autre

Parmi les effets produits (dans la mesure où il n'y a pas calcul ni planification préalable) du parler à plusieurs, l'émergence progressive des genres narratif et argumentatif (d'une ébauche à des configurations plus élaborées), résultant d'un jeu de rôles et de places discursives. Dans un mouvement de convergence les enfants ont appris à garder un thème et à configurer un genre sur plusieurs tours de parole. Des genres jusque là actualisés sous une forme minimale se sont complexifiés, au sens labovien du terme. Les enfants ont doté la marionnette d'une intériorité (Ploum a joué avec ses autos, il "voulait » pas dormir...), l'ont projeté dans le devenir (Ploum qui mange beaucoup « va grandir »). Les processus sont devenus nombreux et divers, mais surtout ce qui a été raconté à partir de la marionnette s'est continué par un récit en Je. L'organisateur dominant de cette hétérogénéité discursive demeurant néanmoins le narratif.

On donne un exemple de l'émergence d'un « raconter à plusieurs », avec cet extrait : (4 Tour, Ludovic, 4,10 ans, est locuteur principal). Le locuteur principal continue le thème. Les interventions de la maitresse contribuent à l'intelligibilité de son discours, elle demande confirmation du dit et suggère un rapport entre les éléments dits, (rapport de temporalité, de logique d'action), composantes nécessaires à la mise en récit.

LUD 4 - <plus fort>i(l) a [roe] dans (l)a forêt à [u]

MAî 14 - ah:::! ben voilà j'entends! il a promené dans la forêt DU LOUP, c'est ça ?

LUD 5 - ouais !

MAî 15 - mm! ah bon! hier soir?

GEO 3 - i(l) s'est pas fait mordre \{brouhaha : on entend des voix\}

MAî 16 - hein? oh demande-lui, je sais pas !

GEO 4 - i(l) s'est pas fait mordre

MAî 17 - ah ben, demande-lui! chais pas

GEO 5 - est-ce qu'i(l) s'est pas fait mordre, quand Ploum, il était promené?

LUD 6 - non

MAT 1 - il a forêt dans [sa]

MAî 18 - hein ?

MAT 2 - la forêt dans [se]

MAÎ 19 - la forêt du cerf?

MAT 3 - $\{X X X X\}$ le la $\{X X\}$ la forêt de de cerf 
MAî 20 - la forêt du cerf, il est allé se promener dans la forêt? mais la forêt du cerf, ça peut aussi être la forêt du loup peut-être!+ hein? \{brouhaha : on entend des voix chut chut chut!

MAT 4 - la poule, (i)l avait la poule dans un sac

MAî 21 - il avait quoi? dans le sac, il avait quoi?

MAT 5 - une poule !

MAî 22 - une poule! ah bon!

MAT 6 - vi! et y a un chien (au)[ i]dans la forêt

MAî 23 - le chien se promenait aussi dans la forêt?

Spontanément, le raconter oral appuie le faire sur des raisons de faire. Dans les prises de parole gérées par les enfants, a prévalu l'intrication des éléments narratifs et des justifications, commentaires, explications. Ces possibles ouverts par le parler à plusieurs dans notre atelier de langage ont été favorisés par le dispositif et le guidage de la maîtresse. La discussion, quant à elle, a été le plus fréquemment induite par le questionnement de la maîtresse, cependant au cours de l'année, quelques grands (4/5 ans et 5/6 ans) ont effectué ce mouvement comme la maîtresse. Ses demandes de définition, d'explicitation ont été suivies de moments où les élèves ont pu confronter, dans l'espace discursif, leurs savoirs et leurs croyances de façon explicite, soit dans la tonalité du conflit soit dans celle de l'accord.

Le narratif aussi bien que l'argumentatif pour exposer des choix subjectifs, des préférences et les justifier, sont d'abord des effets produits (dans la mesure où il n'y a pas calcul ni planification préalable) de l'atelier de langage, puis des effets plus intentionnels du point de vue de la continuité thématique et interlocutive, étayés par les interventions de la maîtresse.

NAB 4 - moi tu sais quand je $s(e)$ rai grand§ ben à chaque fois \{toux d'enfant\} que le p(e)tit déjeuner j'irai à Mac Donald'(s) le midi j'irai à Mac Donald'(s) et même le soir!

MAî 27 - eh ben c'est bien! \{brouhaha : on entend des voix\}

NAB 5 - tous les jours §tous les jours quand j(e) s(e)rai un adulte

ELE - $\quad$ eh ben moi aussi

ELE - ben moi c'est ::::

CHA 3 - quand y a d(e) l'école! $\S^{\circ}$

MAî 28 - quand il s(e)ra un- t'as pas entendu c(e) qu'il t'a dit? quand il sera un adulte mm

NAB 6 - j'aurai pas école!

MAî 29 - tu n'auras pas école si t'es un adulte?

NAB 7 - non!

MAÎ 30 - ben alors moi j(e) suis pas un adulte?

NAB 8 - si mais y a

MAÎ 31 - eh ben alors? et puis j(e) vais à l'école! ttt attends attends attends $\S$ Cécile on est en train de discutailler sur l'adulte

ELE - $\quad$ ah ben moi quand je serai grand $\S$ \{on entend des voix

CHA 4 - on a d(e) l'école quand on va $\{\mathrm{XXX}\}$

$\mathrm{NAB} 9$ - pas toujours

MAî 32 - qu'est-ce (que) tu dis euh Chadia? j(e) compr- j'entends pas d'ici alors on doit pas entendre 
CHA 4 - $\{\mathrm{XXX}\}$ on a d(e) l'école

NAB 10 - pas toujours pasqu'on peut faire euh un autre métier que l'institutrice!

MAÎ 33 - ah oui on peut faire un autre métier que institutrice! oui

GEO 5 - il a aussi l'usine hein

Parallèlement, et de façon naturelle, est apparu dans le dialogue enfantin, un mouvement réflexif. La réflexivité est une capacité transversale à l'oral et l'écrit, même si elle s'exerce différemment dans chacune des situations.

Celle qui émerge dans l'atelier de langage concerne l'apport de nouveau dans le dialogue. Ainsi, dans cet exemple, Geoffrey fait remarquer que l'information a déjà été donnée

ELE - [عkoe] il joué à la pâte à modeler?

GEO 40 - non j'en ai pas j'ai déjà dit

De même Pauline,

PAU 25 - après j'l'ai lavé il était impeccable

NAB 8 - est-ce que tu l'as lavé $§ ?$

PAU 26 - ben oui j'viens d'te parler !

Il arrive que les enfants s'étonnent de la question de la maîtresse (tu sais c'est quoi les moutons ?), demandent le sens d'un mot (il est quoi patate?)

C'est aussi dans le mouvement réflexif que s'est jouée la relation entre les façons de voir des participants et qui a eu comme effet de dessiner des limites de validité au discours de l'autre. C'est le cas dans cet exemple, Geoffrey fait une remarque qui concerne les relations de cohérence :

$1^{\circ}$ Tour, Thomas, 3,5 ans est locuteur principal)

THO 16 - après il l'a sauté dans sa voiture! \{brouhaha : on entend des voix\}

MAî 29 - il a quoi? il a quoi? Thomas!

THO 17 - après il l'a sauté dans sa voiture!

MAî 30 - ah il a sauté dans sa voiture! ah? vous entendez? Ploum a sauté dans sa voiture! \{brouhaha: on entend des voix

MAî 31 - hein? qu'est-ce que tu dis, Geoffrey?

GEO 3 - on n'sait pas si i s'est ach(e)té une voiture !

La réflexivité que l'on peut observer dans l'atelier de langage manifeste la capacité d'écoute des enfants et de prise de distance pour entrer dans le commentaire du dit. Elle est une prise de conscience progressive des normes du dialogue, elle amorce la prise en compte du langage comme objet que l'on peut interroger et étudier. C'est au cours des années de scolarisation ultérieures que la réflexivité continuera à se construire, cette dimension de contrôle est tout à fait nécessaire à l'écrit, et s'exerce alors de façon intentionnelle.

\section{Constitution d'un arrière-fond, mouvement de reprise et mouvement de subjectivation}

Le mouvement de subjectivation qui s'est amorcé dans le dialogue est lié aux pratiques langagières orales et écrites. Le sujet se construit dans et par le discours. 
Le sujet discursif ne préexiste pas à l'interaction, dans le cas de l'atelier, il se dessine dans cette situation, avec ces participants, ce thème et cet entour global qu'est le milieu scolaire.

A l'écrit le sujet (écrivant) qui se construit, est, néanmoins, plus abstrait qu'à l'oral, plus " volontaire » également au sens où l'interlocuteur n'est pas présent et qu'il doit savoir continuer seul.

Le sujet discursif se dessine dans ses modes d'enchaînenements de son discours sur celui d'autrui ou sur le sien propre. Dans l'enchaînement d'un discours sur un autre, catégories, genres, thème peuvent être déplacés. Les déplacements (ou mouvements) dans l'enchaînement ne lui sont pas spécifiques, d'autres peuvent les faire, mais il les fait à sa façon.

Parmi ces mouvements, soulignons l'importance des mouvements associatifs : la singularité d'un locuteur s'observe dans ses mouvements associatifs, à partir de sa "compréhension responsive " du mot de l'autre. L'association éclaire le rapport subjectif à l'objet dont on parle. On mesure ainsi l'importance, du « faire parler» mais aussi « du laisser parler », (François, 1993), du laisser associer.

Le mot, même s'il est partiellement commun, est aussi partiellement différent pour chacun. L'association éclaire le rapport subjectif à l'objet dont on parle, qui par ailleurs est objet d'attention conjointe.

Dans notre atelier, nous observons un double mouvement dans la relation interpersonnelle. Au fur et à mesure du déroulement des séances, les enfants ont été de plus en plus dans une relation de faire comme l'autre, de faire ensemble et dans le même temps à leur manière. Ce double mouvement est le support d'un processus de subjectivation dans le dialogue. Le sujet se construit comme sujet discursif appartenant au groupe tout en construisant sa singularité à travers la mobilité des relations de distance proximité aux discours des autres.

Les mots ont circulé d'un locuteur à un autre, prenant dans chaque nouveau contexte un sens nouveau. Par exemple prendre la place de locuteur principal a été pour certains essentiellement « faire comme ont fait les autres ", reprendre leurs discours ; pour d'autres, sur la base de la reprise, modifier et avoir la possibilité d'innover. Changeant de rôles et de places, dans l'attribution ou la spontanéité, les enfants ont expérimenté des façons de dire qui engagent des façons de se comporter au plan énonciatif, intellectuel et affectif par rapport à l'interlocuteur, au thème et au genre.

Le parler à plusieurs et la répétitivité du dispositif sont à l'origine de deux aspects corrélés, d'une part la création d'un arrière fond partagé à l'origine de routines, constituant une culture commune et la construction d'un horizon d'attente quant à la façon de se comporter discursivement (ce qui n'exclut pas la possibilité d'irruption d'inattendus). L'arrière-fond renvoie à un présent devenu absent, qu'un déclencheur dans la relation du locuteur à la situation re-présente, mais modifie (on peut aussi parler d'accentuation).

Les enfants ont gardé en mémoire, différemment et avec des accentuations particulières ce qui s'est dit dans la (les) séance(s) précédente(s). Les routines ne peuvent pas être observées indépendamment de la prise en compte de l'enchaînement d'un discours sur le précédent (à distance ou à proximité) qu'il s'agisse de celui d'un autre locuteur ou du sien propre.

Par exemple, l'action « manger » (il a mangé) a été fortement posée dès les premières séances, ce qui peut s'expliquer par la mise en place du personnage Ploum, dont en effet l'action «manger des carottes » est devenue un trait spécifique. Cet énoncé a été constamment repris par le locuteur principal, quel que soit l'âge de l'enfant interpellé à cette place. On peut dire que se constitue une routine qui fonctionne comme un prêt-à-dire pour les locuteurs principaux.

Mais les routines ne sont pas stables, elles circulent et sont sans cesse changées, du fait même des types de reprises (reprises partielles, des reprises-modifications ou des reformulations), du fait de la circulation du mot, de l'ouvert des associations personnelles. Par exemple, le thème «manger » initié sur le mode factuel (il a mangé), a été continué sur le mode du processus (s'il mange, il grandit), et c'est alors " grandir » qui a été thématisé (ben toi aussi tu grandis). Cette hétérogénéité est la base d'un maniement complexe du langage. 
Les différences entre les enfants relèvent de l'atelier de langage Ploum, mais aussi de caractéristiques générales. Le locuteur devient «tel » sujet discursif à travers la diversité de ses relations au discours de l'autre (pour une part, le sujet se constitue dans sa relation au collectif), au discours des autres, mais sur la base de ses stratégies préférentielles.

\section{Différences entre les enfants}

Force est de constater les différences entre les enfants lors d'une activité conjointe. Cependant, elles ne relèvent pas toutes de cette situation donnée, mais aussi de caractéristiques générales.

On a choisi d'observer deux enfants, l'un de 4/5 ans, l'autre de 5/6 ans, compte tenu de la problématique de l'entrée dans l'écrit et on a comparé leurs conduites langagières sur le critère de la reprise, le degré de jeu avec le langage et leur capacité à créer des univers discursifs hétérogènes, à jouer avec le présent absent.

\subsection{Ludovic, du faire comme (l'autre) à l'affirmation de soi}

Ludovic (4/5 ans) est devenu un locuteur principal écouté et un auditeur poseur de questions à part entière dans des discussions entre pairs.

Dans les premières séances, il était dans une grande dépendance à l'égard du discours d'autrui, ne faisait que des réponses minimales, mais au troisième Tour, à la reprise des routines se sont mêlés les récits de ses expériences personnelles : dans cet exemple, il initie le thème du «tracteur », récurrent chez l'enfant jusqu'au $4^{\circ}$ Tour

LUD 6 - (i)l a fait (v)élo

MAI 12 - il a fait du vélo oui!

LUD 7 - été [kraktoeu]!

MAI 13 - j'ai rien entendu parce qu'ils font du bruit! ++redis moi!

LUD 8 - [a ete kraktoeu]!

MAI 14 - ah! il est allé au tracteur alors qu'est-ce que c'est que ce tracteur dont tu me parles toujours?

LUD 9 - le [ro]

\section{MAI 15 - ah:: c'est le gros tracteur! d'accord!}

Ses sources discursives se sont diversifiées, il a intégré à ses énoncés un thème culturel typique de l'imaginaire enfantin « le loup dans la forêt», Ploum devient un promeneur dans « la forêt du loup », mais « ne se fait pas mordre » (voir extrait reproduit plus haut).

Ses modes de participation se sont considérablement augmentés, il est intervenu pour donner des précisions lexicales (pour désigner Ploum), faire des remarques dubitatives «dans l(e)sac? 》, des commentaires méta-discursifs, «ça fait beaucoup d(e)temps! » et aussi des commentaires appréciatifs : « est [z]oli comme ça».

La reprise a d'abord été un mode privilégié lui permettant de devenir locuteur. Il s'est d'abord fait « comme l'autre» en enchaînant, par exemple, sur l'énoncé précédent par un «moi aussi ». Il a pu ultérieurement manifester son désaccord, "pas moi » répond-il à celui qui affirme vouloir parfumer Ploum, et surtout il est entré dans l'associatif, à partir du discours des autres, enchaînant avec " peinture » sur celui qui parle de «dessin », et a donné son point de vue en se posant en tant que « je »:

NAB- @ moi après j'ai/j'suis tombé/ puis main(te)nant j'y arrive aux patins à roulettes !

$$
\text { LUD- moij'aipeu(r) } \S \S
$$


L'évolution de Ludovic nous permet d'apprécier les effets du parler à plusieurs et son puissant pouvoir de construction de soi.

\subsection{Geoffrey, le plaisir de parler et de créer}

Les conduites langagières de Geoffrey (5/6 ans) sont largement dépendantes de sa place discursive : quand il est locuteur principal, il reprend mais surtout modifie les actions rituelles de Ploum, et monologue dans le dialogue pour raconter et construire un univers du discours, entre reprise d'expérience personnelle et fiction.

Dans ce premier exemple, il reprend un thème récurrent «laver Ploum» mais pour le singulariser en rapprochant le sens de bain « de rendre propre» de celui des jeux d'eau (plonger) en affinité avec le mot «piscine »

GEO 10 - si ! pasque moi j'l'ai lavé, j'l'ai lavé dans ma piscine!

MAî 37 - ah tu l'as lavé dans ta piscine

GEO 11 - §ouais j'l'ai jeté en l'air et ....

MAî 38 - alors tu dis c'est parce que j'l'ai lavé aussi, il devrait être propre donc Ploum

GEO 12 - ben non! avec du savon!

THI 3 - moi [ [i] piscine ! a été à ma piscine !§

GEO 13 - et pis ça a pas disparu! ça cache une tache de thé

MAî 39 - aha! c'est une tache de quoi?

GEO 14 - de thé

MAî 40 - ah! bon \{brouhaha : on entend des voix\}

GEO 15 - $\{\mathrm{XXX}\}$ en plus c'est une grande piscine, tout au fond! après j'l'ai jeté en l'air, il a fit un plongeon!

LUC 1 - moi j'ai une piscine !

Et dans cet exemple, il crée un monde de fiction, en prétendant avoir doté Ploum de la parole au moyen de piles :

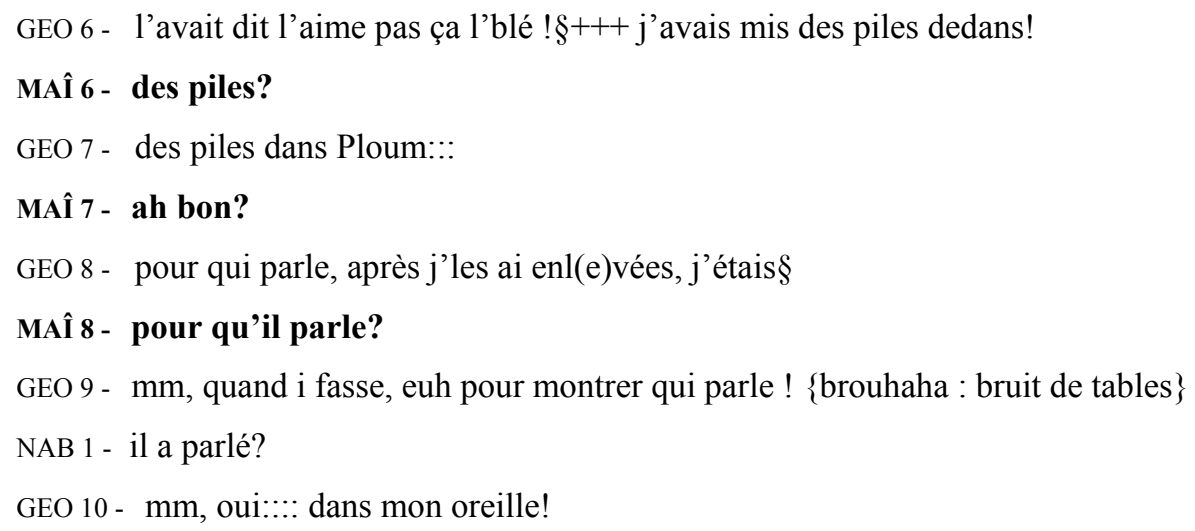

Il sait composer un récit complexe en enchaînant des énoncés hétérogènes, et donner des précisions lexicales quant à l'espace, au temps, au monde familial. Sa spécificité est le mélange des mondes : réel, 
fictif et ludique, le va-et-vient entre générique-particulier et entre pour de vrai/ pour de faux, corrélé avec familier/étrange.

Il se comporte également comme un enfant très préoccupé d'établir des relations, de définir les aspects du bien, du mal, du normal, de l'admissible, de justifier, d'expliciter : par exemple, lorsque les pairs s'étonnent de la propreté de Ploum, "j'fait prendre un bain c'est normal» ou encore quand on lui fait remarquer que les poils de la marionnette ont été coupés "non! juste à cause de mon savon il lui a rétréci (les poils)»

Quand il n'est pas locuteur principal, il suit l'adulte, il est dans une relation de coopération avec la maitresse avec, une forte implication de soi, des commentaires subjectifs, des jugements de valeur.

Les effets du parler à plusieurs ne concernent pas seulement le cognitif mais aussi la construction de soi.

\section{Pour conclure}

Le développement et l'appropriation des conduites langagières orales et l'entrée dans l'écrit sont partiellement des caractéristiques générales qui ne sont pas propres à l'école, comme le dialogue des sémiologies, le rapport dialogique, l'espace transitionnel, mais aussi partiellement développés et favorisés par l'institution scolaire.

Le plaisir des jeux langagiers est favorable au développement des conduites langagières aussi bien orales qu'écrites et sans nul doute, l'atelier de langage organisé autour d'un objet d'attention conjointe attrayant a eu cette capacité. Par la souplesse de son dispositif, il s'est montré propice à un mode de participation diversifié de la part des enfants, autour de Ploum, à la fois commun et singulier pour chacun.

Notre atelier permet également de préciser l'importance du dialogue et du dialogisme.

L'analyse du déroulement des échanges sur la longue durée a montré le développement et le mode d'appropriation des conduites langagières. Tout au long du dialogue, les enfants ont pu faire, à plusieurs, l'expérience de l'hétérogénéité discursive, base d'un maniement complexe du langage.

En ce sens, situation qui se répète et constitution d'un groupe, deux contraintes de la situation scolaire, constituent des éléments dont on doit tenir compte pour évaluer les conditions du développement langagier des enfants.

Le parler à plusieurs est un moment capital dans la construction de soi, de l'altérité et de sa culture qui semble indispensable à la possibilité de devenir un sujet écrivant, capable de prendre une position énonciative et de continuer seul. La répétitivité, en tant que modalité du dispositif, est en affinité avec la constitution d'un horizon d'attente et l'élaboration d'un arrière fond (discursif, pathique et doté de valeurs) qui fonctionne à la fois comme cadre dans lequel les énoncés font sens et comme culture partagée commune. L'atelier a permis de mettre en lumière l'importance du groupe comme médiateur et l'hétérogénéité des mouvements de reprise modification, selon la place discursive, le parler dans le thème ou hors du thème Ploum, le genre et le monde. Le mouvement de reprise modification de l'arrière fond culturel ainsi élaboré collectivement dans le dialogue permet d'initier un jeu avec le présent absent, qui est prégnant dans l'écrit.

Cependant, chaque enfant a, dans une séance et dans la succession des séances (sur la longue durée), sa façon de s'investir dans le parler à plusieurs, sa façon de reprendre le discours de l'autre. La place discursive introduit une variable très importante. Tous les enfants ne développent pas les mêmes capacités. Disant cela, nous voulons souligner le fait que chacun a ses usages langagiers préférentiels (ses thèmes et ses genres, ses modes d'enchaînements sur le discours de l'autre) en fonction de ses affects et de son histoire. L'atelier de langage (ou toute autre situation d'enseignement apprentissage langagier) n'a pas comme objectif d'amener les enfants à parler tous de la même façon, ou à parler comme l'adulte, mais de leur permettre en tant que groupe, membre du groupe, et tel enfant, de jouer avec le langage, d'expérimenter les changements de registre discursifs et sémiotiques, de construire des mondes entre réel et fiction, de comparer des rapports de sens, ensemble de dispositions favorables à l'entrée dans l'écrit. 
Didactique et enseignement, français langue maternelle, français langue seconde DOI $10.1051 / \mathrm{cmlf} / 2010155$

\section{Bibliographie}

Bakhtine, M. (1984). Esthétique de la création verbale. Paris : Gallimard.

Bakhtine, M. [V.N. Volochinov] (1929/1977). Le Marxisme et la philosophie du langage. Essai d'application de la méthode sociologique en linguistique. Paris : Minuit

Brigaudiot, M. et Danon-Boileau, L. (2002), La naissance du langage dans les deux premières années, Paris : PUF.

Bruner, J.S. (1983). Comment les enfants apprennent à parler. Paris : Retz.

Colletta, J.M. (2001), Quand les enfants racontent leur expérience avec les mots, la voix et le corps, Revue Histoires de vie, 2, 27-33 et 101-109.

Fabre, C. (1990). Les brouillons d'écoliers. Grenoble : Ceditel

François, F. (1993). Pratiques de l'oral, Dialogue, jeux et variations des figures du sens, Paris : Nathan.

Froment, M. et Leber Marin, J. (2003). Analyser et favoriser la parole des petits. Un atelier de langage en maternelle. Paris : ESF

Goody, J. (1977). La raison graphique. Paris : Minuit.

Halté, J.F. et Rispail, M. (éds) (2005). L'oral dans la classe, Paris : L'Harmattan

Kügler, M. (2000). L'échec en lecture handicape-t-elle moins les filles que les garçons ?, La nouvelle revue de l'AIS, 9, $108-121$

Morgenstern, A. (2009). L'enfant dans la langue. Paris : Ophrys

Nonnon, E. (1996). Interactions et apprentissages, Le français aujourd'hui « Interactions : dialoguer, communiquer », $113,55-63$

Pouëch, F. (2001). Effets des jeux langagiers de l'oral sur l'apprentissage de l'écrit. Paris : L'Harmattan

Préneron, C., Meljac, C. et Netchine, S. (1994). Des enfants hors du lire. Paris : Bayard

Vygotski, L.S. (1934/1997). Pensée et langage. Paris: La Dispute.

Winnicott, D. W. (1971/1975). Jeu et réalité, l'espace potentiel. Paris: Gallimard

\footnotetext{
1 « Maîtrise de la langue »: L'emploi qui est fait de cette expression dans les textes officiels est porteur de malentendus. Il entretient une confusion entre langue et discours.

${ }^{2}$ L'enfant acquiert le langage dans le milieu familial en interagissant et dialoguant avec les adultes qui l'entourent, sur la base d'une communication non linguistique. Bruner (1983/1987) développant la notion de format ou scénario d'interaction, et plus récemment des recherches en psycholinguistique et didactique (Brigaudiot et Danon Boileau 2002, Morgenstern, 2009) se sont attachées à montrer la naissance du langage dans l'environnement familier de l'enfant.

${ }^{3}$ L'analyse de cet atelier de langage a été publiée en 2003, sous le titre Analyser et favoriser la parole des petits. Un atelier de langage en maternelle.

${ }^{4}$ Le Tour est ici l'unité de passage de l'effectif de la classe. La majuscule est la marque de la distinction entre Tour de passage et tour de parole.

${ }^{5}$ Les « bombardements de questions » sont des moments au cours desquels les enfants, tous ensemble et non alternativement, posent des questions sans attendre de réponse, donnant naissance le plus souvent à un brouhaha.

${ }^{6}$ Conventions de transcription : Transcription orthographique. Le chiffre qui suit les trois premières lettres du nom rend compte du nombre de tours de parole de ce locuteur, à ce moment de la séance. Le signe + indique une pause, dont la longueur est proportionnelle au nombre de croix ; le signe § signale un enchaînement rapide ; le soulignement indique un chevauchement; entre parenthèses, les phonèmes non prononcés par les locuteurs ; entre crochets $(<\ldots>)$, des indications portant sur le non verbal. En gras les interventions de la maîtresse.
} 